Revista Científica de FAREM-Estelí

Medio ambiente. tecnología y desarrollo humano

Año 10 | Edición especial: artículos de revisión documental

ISSN: 2305-5790

https://rcientificaesteli.unan.edu.ni

DOI: https://doi.org/10.5377/farem.v0i0.11614

\section{Dimensiones para la sustentabilidad de los sistemas de producción agropecuarios excluidos de la agroecología}

\section{Dimensions for the sustainability of agricultural production systems excluded from agroecology}

\section{Darwin Ráudez-Centeno}

Centro de desarrollo tecnológico, San Isidro, Instituto Nicaragüense de Tecnología agropecuaria INTA-Nicaragua.

https://orcid.org/0000-0002-2599-6743

darwincenteno19@yahoo.es

\section{Jairo Emilio Rojas Meza}

Doctor en Ciencias en Estrategias para el Desarrollo Agrícola Regional (Ciencia y Tecnología). Docente Investigador en la UNAN-FAREM-Matagalpa.

https://orcid.org/0000-0003-1980-1599

jrojas_12@yahoo.com

\section{RESUMEN}

El propósito de este artìculo es profundizar en los distintos enfoques teóricos para la sustentabilidad de los sistemas de producción agropecuarios excluidos de la agroecología, se parte de la hipótesis de que las magnitudes que se mencionan teóricamente en esta ciencia son incompletas para expresar el alcance y el sostén de la productividad agrícola moderna y sobre todo lograr la seguridad alimentaria, por lo cual se proponen algunas extensiones que complementen las ya planteadas y se tomen como elementos básicos para dicha ciencia, la concepción filosófica y teórica para argumentar una ampliación de las dimensiones y de su aplicabilidad se origina en autores que son expertos en ciencias económicas, ecológicas y agronómicas, el ensayo sugiere ampliar más el alcance medible de esta asignatura por eso se proponen sustentable, adaptativa, pluralidad, mercado, universalidad. La medición de estas nuevas facetas para la integralidad de esta disciplina, tomando un enfoque de universalización, aplicación y entendimiento de la misma porque la asociación de estos principios es lo que hará de la disciplina una ciencia creíble y práctica.
RECIBIDO

$26 / 03 / 2021$

ACEPTADO

19/052021
PALABRAS CLAVE

Aplicación; ciencia; disciplina; ecológica. 
KEYWORDS

The purpose of this article is to deepen in the different theoretical approaches for the sustainability of agricultural production system excluded from agroecology, the Application; science; hypothesis is based on the assumption that the quantities theoretically mentioned in this science are incomplete to express the scope and support of modern agricultural productivity and above all to achieve food security, the philosophical and theoretical conception to argue for an extension of the dimension and their applicability originates in authors who are expert in economics, ecological, and agronomic science, the article suggest to extend more the measurable scope of this subject, that is why sustainability, adaptive, plurality, market and universality are proposed. The measurement of these new facets for the integrality of this discipline, taking an approach of the universalization, application and understanding of it because the association of these principles is what will make the discipline a credible and practical science. 


\section{INTRODUCCIÓN}

La agricultura convencional viene afrontando una profunda crisis de producción, debido principalmente a su carácter de fertilización bajo agro tóxicos para poder mantener la capacidad productiva de los sistemas (Tranquilli, 2015).

Este tipo de práctica agrícola, tiene influencia directa en los sistemas de producción, dado que si no existe una alta fertilización no se logra obtener cantidades necesarias de alimentos para el sustento de una población cada vez más creciente y demandante.

Como respuesta a esta problemática, se da el surgimiento de la agroecología, la cual nace como un nuevo enfoque científico que plantea una nueva forma de pensar y hacer agricultura, y ofrece las bases científicas y metodológicas para avanzar hacia una agricultura sustentable. (Dalgaard et al, 2003).

Sin embargo, se considera que las dimensiones propuestas por la agroecología no son suficientes para lograr la sustentabilidad de los sistemas de producción. Es por eso que en el presente ensayo se plantea como objetivo principal analizar las dimensiones sugeridas por la agroecología y proponer una serie de dimensionales integrales, que fomenten la incorporación de las ciencias modernas y logren aportar a la sustentabilidad del sistema productivo.

De acuerdo con Nosty (2009), la defensa del medio ambiente y el desarrollo sostenible se han convertido en objetivos universales ya que esta acentúa las contradicciones de un modelo de sociedad basado en la primacía de los intereses del sistema productivo. Esto implica una relación armoniosa tanto de los seres humanos con el medio ambiente, como la relación en la sustentabilidad de los sistemas productivos.

La sustentabilidad de la agricultura actualmente se encuentra en un gran riesgo, dado que la mayoría de los modelos de cambios de climas, predicen que los daños serán de forma desproporcionada para los pequeños agricultores, (Altieri y koohafkan, 2008).

Estas alteraciones climáticas primeramente están atribuidas al mal manejo de los sistemas productivos y segundo ala desproporcionalidad de un equilibrio ecológico, el cual contempla obtener altos rendimientos, pero con altas aplicaciones de fertilizantes y pesticidas sintéticos cuyo contexto está basado en la agricultura convencional.

La integración y aplicación que pregona la agroecología, no son elementos suficientes para definir la sostenibilidad del complejo de producción, pues 
consideramos que hay elementos importantes que están marginados en los principios diseñados y sostenidos en esta ciencia.

Para profundizar, ¿cuáles serían aquellos enfoques excluidos en la agroecología para la sostenibilidad de la producción agrícola? en este artículo realizamos una revisión teórica de esta materia y en particular los sustentos que derivan de dicha ciencia. La base metodológica de este artículo es la investigación documental, utilizando bibliografía en físico y digital.

El artículo se despliega en tres partes: en la primera parte, se aborda el sustento conceptual de esta disciplina, sus postulados y principios, ya que contextualmente se considera una ciencia que revoluciona el rendimiento sustentable y que muchos países han puesto en práctica para resolver la problemática de la debacle de la agricultura convencional.

En la segunda parte se abordan las propuestas teóricas y conceptuales de la sostenibilidad de la productividad y los aportes de esta materia, en esta parte se vaticina encontrar confluencias y discrepancias entre ambos postulados.

En la tercera parte se precisan un conjunto de principios que podrían contribuir a reforzar esta ciencia para el sostén de los sistemas de producción agrícola y que podrían incluirse en los principios ya definidos por la agroecología.

\section{MATERIALES Y MÉTODOS}

Para lograr obtener información básica sustentada en datos científicos, se realizó una revisión sistemática de revistas científicas indexadas y dedicadas a las publicaciones de artículos, libros y memorias en línea sobre la agroecología.

El principal buscador en línea fue google académico, donde se ubicaron artículos publicados en revistas de sostenibilidad agrícola, revista sociedad y recursos naturales, también se revisaron las bases de datos de: Scielo, latindex y redalyc. En total se localizaron, 17,300 estudios, de los cuales solo 30 artículos y 2 libros fueron los más relevantes. Los artículos y libros seleccionados, fueron leídos detenidamente, y se precisan los aspectos de relevancia que aportaron a los planteamientos y la discusión que se presenta en este artículo. 


\section{RESULTADOS}

La agricultura es una de las actividades humanas más importantes, pareciera que ha existido desde tiempos inmemoriales y va a existir por siempre. Sin embargo, durante la mayor parte de su historia en el planeta, la especie humana no practicó la agricultura: se adecuó a la naturaleza, viviendo de la caza y la recolección. Es decir, que durante la mayor parte de la historia de la humanidad el ser humano se alimentó, se vistió y satisfacía sus necesidades básicas sin practicar la agricultura (Sarandon y Flores, 2014).

La agricultura nace de la necesidad de alimentarse, es decir que a medida que los tiempos y la ciencia avanzaban se fue haciendo ya una práctica necesaria para la sobrevivencia, sin embargo, la aplicación irracional de técnicas y herramientas de modelos intensivos ha propiciado un camino incorrecto de la agricultura.

Lo aportado por Sarandon y Flores (2014), el cual define que, si bien es cierto que la tecnificación de la agricultura ha incrementado, a través de un mayor rendimiento (por unidad de área) de los cultivos, la producción de alimentos en el mundo, pero no es menos cierto también, que esto ha estado basado en el uso de dosis masivas de insumos costosos.

Efectivamente, este criterio que la agricultura, no solo ha estado en detrimento de uso de dosis masivas de insumos costosos si no también el alto costo que implica cambiar y adecuar la produccion para obtener buenos rendimientos.

A que me refiero con esto, que a medida que se aplican insumos costosos el cultivo se adecua a esta forma de hacerlo producir y una vez que no recibe estas altas cantidades de insumos, simplemente no produce y si logra producir lo hace en cantidades muy pequeñas que no son rentables económicamente, entonces tenemos que caer a la intensificación para poder producir.

Esta intensificación en el uso de insumos químicos en la agricultura, junto con el uso inapropiado de ciertas tecnologías, provocaron impactos que perjudicaron, tanto a los recursos propios de la agricultura, atentando contra su capacidad productiva, como a los recursos globales o de otros sistemas (Mc Ginn, 2000b).

\section{Contaminación por plaguicidas}

Según el programa de Naciones Unidas para el Medio Ambiente (PNUMA, 1990), los plaguicidas utilizados en la agricultura pueden tener efectos negativos sobre la población relacionada directamente con las actividades agropecuarias y también con la salud de los consumidores de los productos provenientes de las mismas. 
Los plaguicidas sintéticos son tóxicos bajo cualquier circunstancia y más aún cuando se desconoce técnicas adecuadas de aplicación, no descartaremos que son precisamente parte de la solución a los problemas de la agricultura moderna. Sin embargo, debe de haber un equilibrio lógico en su aplicación para obtener resultados positivos no solo en la producción si no en el medio ambiente.

En la actualidad casi no se concibe la posibilidad de una agricultura sin un importante aporte de los agroquímicos, fundamentalmente pesticidas. Como señala Mc Ginn (2000a) "la agricultura moderna tiene una seria dependencia de agroquímicos - una adicción a los pesticidas". La aparición de los plaguicidas de síntesis abrió una nueva era en el control de las principales adversidades bióticas que limitaban la producción de los cultivos.

Entonces basado en esto podemos considerar que, actualmente se reconoce que el paradigma de la "Revolución Verde" está agotado y superado desde hace tiempo y que no se tuvieron en cuenta las externalidades ambientales negativas generadas por el uso intensivo de fertilizantes y agroquímicos para controlar plagas y enfermedades (IICA, 1999). El problema más claro es el daño ambiental (IICA, 2012).

\section{Dimensiones ecológicas sociales y económicas de la crisis de la agricultura industrial moderna}

Crisis ecológicas: Según Tribi (1994), la demostración más clara de crisis ecológica es la estabilización de los aumentos de rendimientos productivos, en algunos lugares, los rendimientos están disminuyendo (Hewitt y Smith, 1995). No solo es un rendimiento productivo basado en términos agrícolas, si no en procesos ecológicos, que implica esto que el ambiente donde se producía determinado cultivo hoy ya no se puede, aunque se apliquen grandes cantidades de pesticidas y fertilizantes sintéticos.

Algunos creen que los rendimientos se están estabilizando, porque se está acercando al máximo potencial de rendimiento de las variedades actuales y, por lo tanto, se debe aplicar la ingeniería genética a la tarea de rediseñar las especies de cultivos que se produce actualmente.

Sin embargo, por otro lado, tenemos el pensamiento de los agroecológos que creen que la estabilización se debe a la constante erosión de la base productiva de la agricultura a través de prácticas insostenibles (por ejemplo, Hewitt y Smith 1995; Altieri y Rosset, 1995).

Crisis económicas: la realidad es que los agricultores se han visto cada vez más atrapados en una contracción de costos y precios, por lo cual los crecientes costos de la tecnología agrícola moderna han absorbido constantemente cualquier aumento en los ingresos agrícolas. Por lo cual Rosset (1997), define que si existe un modelo alternativo tendrá que reducir

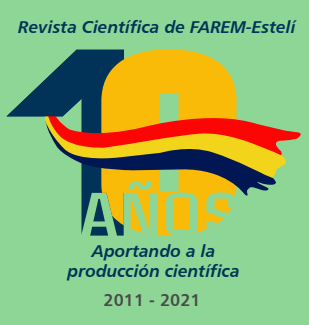


drásticamente la dependencia de costosos insumos no agrícolas para ayudar a los agricultores a salir de esta crisis.

Crisis sociales: Una crisis económica directamente te causara un impacto social, dado que los productores al no tener rentabilidad en sus producciones para el sustento de sus familias, pues terminan trabajando a otras personas o emigrando de sus lugares y empobreciendo más un país en desarrollo.

No obstante, no solo hablamos de crisis, ecológica, económica y social, sino que también hay que mencionar que existe una crisis cultural, dado que ha sido costumbre establecer cultivos con altos rendimientos y mejorados genéticamente, y olvidando los cultivos tradicionales.

Entonces, lo que se necesita y se requiere, es rescatar toda la esencia cultural productiva, que poseíamos antes, si bien es cierto no teníamos tantos problemas de plagas y enfermedades en la agricultura como ahora. Pero, hay que reflexionar, que el ser humano ha sido el principal causante de la debacle agrícola, y por tanto, se debe asumir la responsabilidad de cambiar el rumbo.

También es necesario mencionar que hay una crisis de conciencia, hace falta conciencia de que la agricultura extensiva convencional es dañina, y aunque se logra producir más, no se puede obviar que existen afectaciones, a tal grado de que existen suelos que no son fértiles y que su fin es convertirse en suelos desérticos inútiles para la agricultura.

\section{La Agricultura Sustentable: Un desafío complejo}

El concepto "oficial" y generalmente aceptado de "Desarrollo Sustentable" es el acuñado por la comisión Brundtland como "aquél que permite la satisfacción de las necesidades de esta generación sin comprometer la satisfacción de las necesidades de las generaciones futuras" (CMMAD, 1988).

Sin embargo, Sarandón et al., (2006.) no están de acuerdo con esta definición y proponen que "Una Agricultura Sustentable" es aquella que mantiene en el tiempo un flujo de bienes y servicios que satisfagan las necesidades alimenticias, socioeconómicas y culturales de la población.

Generalmente, el concepto habitual sobre la agricultura sustentable está estrictamente ligada a la armonía con la naturaleza, lo que significa que no puede sacrificar ni la seguridad alimentaria de una población en crecimiento, ni poner en riesgo la naturaleza que es donde nacen los alimentos y esto únicamente se logra con cambios de paradigmas en el pensamiento, aprendemos a desaprender, y cambiamos el rumbo de la agricultura actual. 


\section{El enfoque agroecológico: el camino necesario}

Altieri (1987), menciona que es necesario entonces, un nuevo paradigma que intente dar soluciones novedosas partiendo de la consideración de las interacciones de todos los componentes físicos, biológicos y socioeconómicos. Este nuevo enfoque es la agroecología, que ha sido definida como el desarrollo y aplicación de la teoría ecológica para el manejo de los sistemas agrícolas, de acuerdo a la disponibilidad de recursos.

Como se mencionó, la ciencia agroecológica, surge como un nuevo enfoque científico que plantea una nueva forma de pensar y hacer agricultura, y ofrece las bases científicas y metodológicas para avanzar hacia una agricultura sustentable (Altieri \& Nicholls, 2012; Sarandón \& Flores, 2014b; López García, 2015; Wezel \& Soldat, 2009; Perez-Rosado, 2005; Francis et al., 2003).

\section{Dimensiones consideradas en la agroecología}

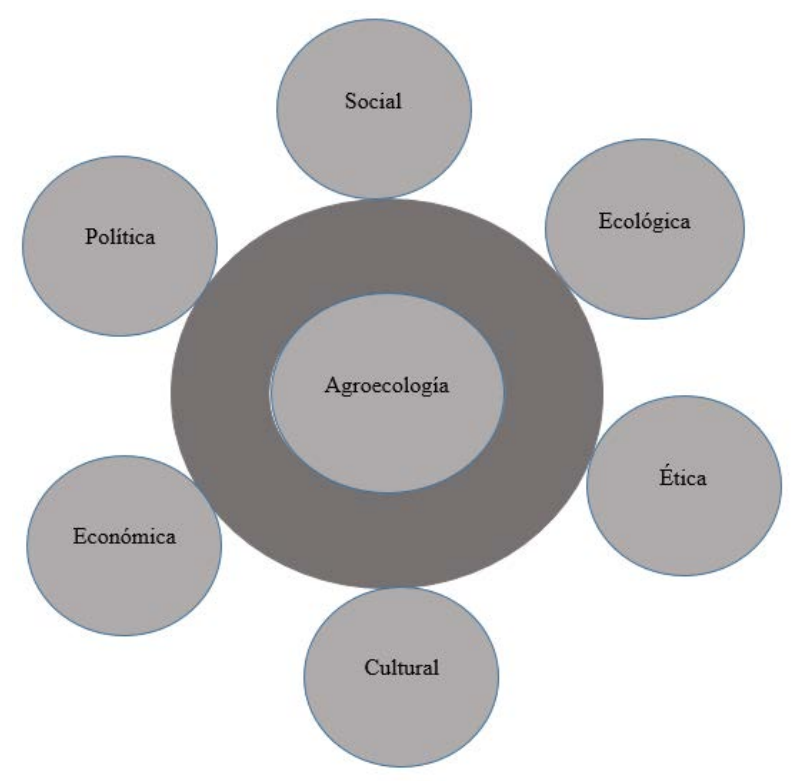

Gráfica 1: Dimensiones de la ciencia agroecológica (Sarandon y Flores, 2014)

La disciplina agroecológica, parte de una serie de premisas metodológicas para desarrollar, integrar y mejorar las prácticas que respondan a sus principios (Guzmán et al., 2000): Un enfoque holístico y sistémico y una mirada multidisciplinaria para la sustentabilidad.

La sustentabilidad debe ser vista como una búsqueda permanente de nuevos puntos de equilibrio entre estas diferentes magnitudes, que pueden ser conflictivas entre sí en realidades concretas (Caporal \& Costabeber, 2004a). 
Entre estas magnitudes se menciona: la social la cual define una distribución más equitativa (tanto de la producción como de los costos), la cultural: dado que esta ciencia entiende que la intervención sobre los agroecosistemas debe considerar los valores y saberes locales de las poblaciones rurales.

Posteriormente existe un alcance ecológico: la agroecología busca la conservación y rehabilitación de los recursos naturales a nivel local, regional y global utilizando una perspectiva holística y un enfoque sistémico. Una magnitud económica: la cual busca el logro de un beneficio que permita cubrir las necesidades económicas del productor y su familia.

De igual forma la agroecología plantea un alcance político: el cual tiene que ver con los "procesos participativos y democráticos que se desarrollan en el contexto de la producción agrícola y del desarrollo rural."

Caporal \& Costabeber, (2004 a) también menciona una dimensión ética: la agroecología insiste en la necesidad de componer un nuevo vínculo moral, que incluya el respeto y la preservación del medio ambiente no sólo para éstas, sino también para las futuras generaciones.

La agroecología con todos sus componentes, surge como una alternativa de solución a los problemas planteados por la agricultura convencional dado que es una ciencia que contempla una visión integral para sacar adelante la agricultura moderna.

\section{La ciencia agroecológica como movimiento populista}

Las técnicas agroecológicas, basadas en la imitación de los ciclos naturales, reducen la necesidad de insumos y ayudar a crear ecosistemas en crecimiento que fomenten formas más regenerativas de producir alimentos con la naturaleza FAO, (2014).

Es importante destacar que esta ciencia, como alternativa a los sistemas agroalimentarios actuales se remonta a su origen como expresión de resistencia a la agricultura industrial (Guzmán, 2013) y la revolución verde (Gliessman, 2013). De la misma manera que es considerada una ciencia populista es precisamente porque emana del conocimiento ancestral de los agricultores.

\section{Agricultura intensiva sostenible versus la disciplina agroecológica}

La ciencia y la tecnología moderna son componentes esenciales de la agricultura intensiva sostenible, así mismo su objetivo es aumentar la productividad agrícola, reducir los impactos agrícolas negativos en el medio ambiente y mejorar la producción (Kershen, 2006). Todos los aspectos parten desde una sola vía, la sostenibilidad como se denota en el cuadro 1. 
Cuadro 1 vías agrícolas sostenibles, propuesto por Mockshell (2018).

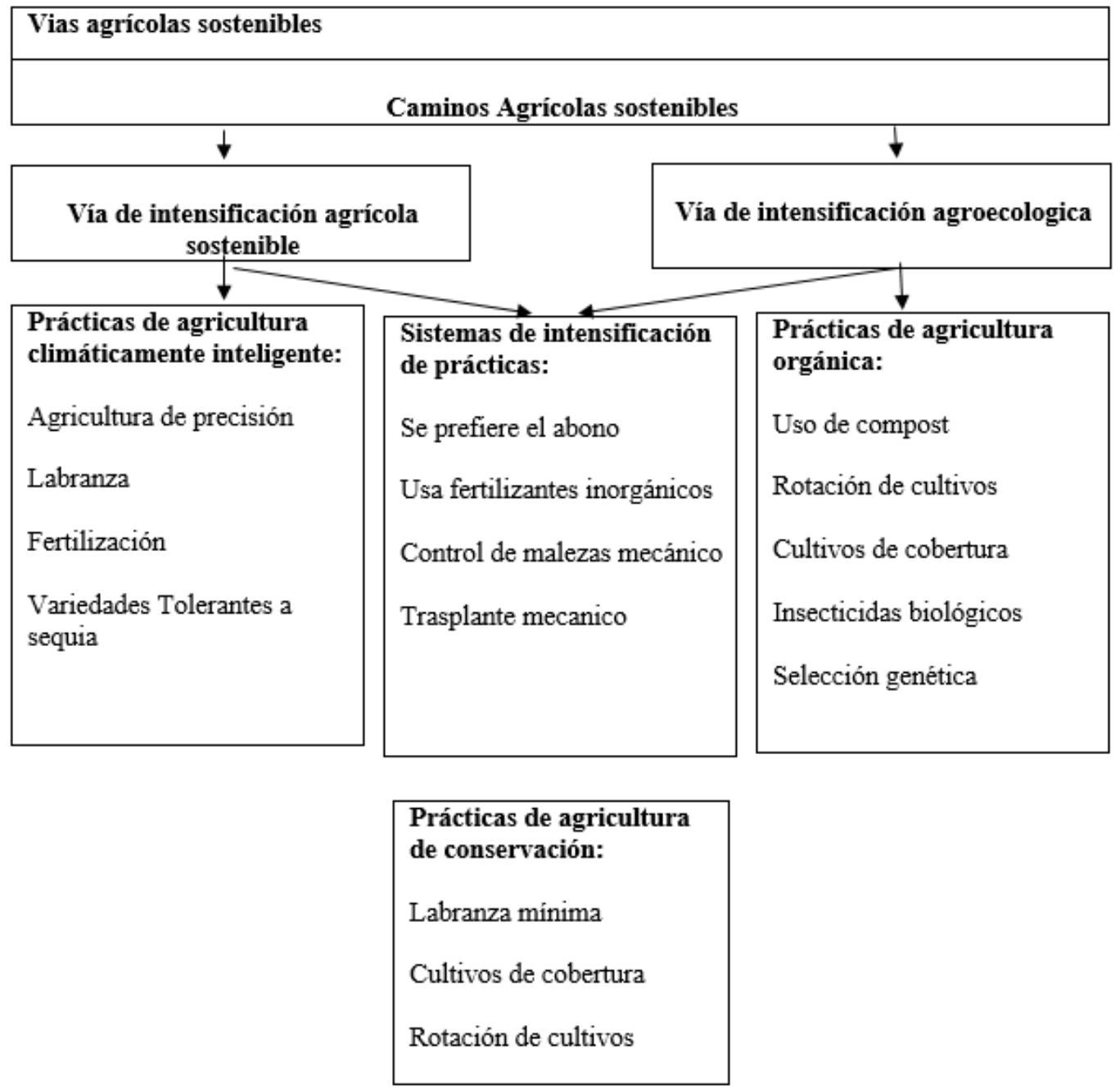

De Schutter (2010), define que la agroecología es una ciencia y un conjunto de prácticas. Como ciencia, esta disciplina es la "aplicación de la ciencia ecológica al estudio, diseño y gestión de agro ecosistemas sostenibles.

Evidentemente la agroecología conjuga la ecología con el sistema agropecuario, y que implica esto, una inclusión total de los factores naturales y humanos bajo una sola dirección la produccion sustentable.

Sin embargo, Garnet y Gofray, (2012), comentan que los objetivos planteados entre la agricultura intensiva sostenible y las ciencias agroecológicas, tienen mucho en común, pero en los métodos y prácticas, sin embargo, en el desempeño funcional para el futuro de la agricultura estas dos visiones son bastante dispares.

Porque mencionamos que son impares, si bien la agricultura intensiva sostenible puede adoptar y ser complementaria a la mayoría de las prácticas 
agroecológicas, los defensores de estas prácticas son explícitos en que esta disciplina rechaza la agricultura intensiva sostenible.

(De Schutter y Vanloqueren, 2011). En particular rechaza el énfasis en las ciencias modernas como la biología, la química y la tecnología moderna; como los cultivos transgénicos y los insumos manufacturados.

En este sentido se considera que la agroecología debería de incluir aportes de los avances de las ciencias modernas, conjugar ambos conocimientos y encaminarlos a la práctica, para lograr un equilibrio en la agricultura. La agroecología debe sustentar cultivos altamente productivos, pero sin necesidad de tantos insumos sintéticos, ese es el aporte que debe de unificar a la agricultura intensiva sostenible con la agroecología.

Sin embargo, los defensores de esta nueva ciencia, describen burlonamente la agricultura intensiva sostenible como una cultura moderna de tecnociencia posterior a la segunda guerra mundial que adopta un camino centralizado de arriba hacia abajo para el alivio de la pobreza y el hambre. Estos mismos proponentes apoyan la agroecología como una práctica que enmarca la pobreza y el hambre como social, conductuales y políticos, y enfatiza un camino localizado de abajo hacia arriba para el alivio de la pobreza y el hambre (Quist et al., 2013).

Keller, (2013), resalta que, la rama agroecológica según la definen muchos de sus proponentes excluye explícitamente la ciencia moderna, la tecnología moderna y una orientación productiva para la agricultura. De hecho, muchos agricultores convencionales ya utilizan prácticas agroecológicas, aunque es más probable que los agricultores las llamen prácticas de conservación.

De igual forma se acuña que la agricultura intensiva sostenible es tecnológicamente neutra. La agroecología con sus exclusiones a las ciencias modernas no presenta la neutralidad en la agricultura. Sin embargo, la agroecología con sus postulados aun no complementa la necesidad real y práctica de la agricultura, porque como es denominada una ciencia integral debe de considerar las ciencias modernas en su conjunto.

Así mismo, la agroecología no es idéntica a la agricultura orgánica, los defensores de dicha ciencia invocan constantemente a la agricultura orgánica como el camino a seguir para la producción agrícola (Quist et al., 2013).

Es una realidad que la agroecología es el camino para obtener una agricultura equilibrada, sin embargo, tiene que satisfacer los criterios integrales de la produccion, sin exclusión de ninguna índole, ni siquiera aun de las ciencias modernas. 


\section{Propuesta dimensional de inclusión en la agroecología, para la sustentabilidad de los sitemas de producciones agropecuarios}

El razonamiento que afirma este planteamiento, de incluir algunas mediciones que no incluye la ciencia agroecológica, se basa en que las magnitudes planteadas por esta ciencia son insuficientes para resolver la crisis y los problemas demandados por la agricultura actual.

La transformación de la agricultura por medio de esta disciplina, desde el punto de vista de los autores de este artículo, es un proceso engorroso y de amplio rango de dominios, ya que esta materia como ciencia multidisciplinaria y de grandes conjugaciones, se aleja conscientemente de la universalización. Por ello, las decisiones analíticas universales porque existen contexto y características diferentes.

Los rangos que actualmente contempla dicha materia (son: ecológicas, económicas, sociales, culturales, políticas y éticas). Esto refleja una gran integración desde un punto de vista teórico, pero desde un punto de vista práctico sometemos a consideración cinco extensiones que son: sustentable, adaptable, pluralidad, conexión con el mercado y sobre todo universalidad. Cada una de estas propuestas representa una necesidad para la sostenibilidad del modelo agroecológico productivo y sobre todo la sostenibilidad de la agricultura actual.

\section{Gráfica 2: dimensiones integrales de la agroecología (elaboración} propia)

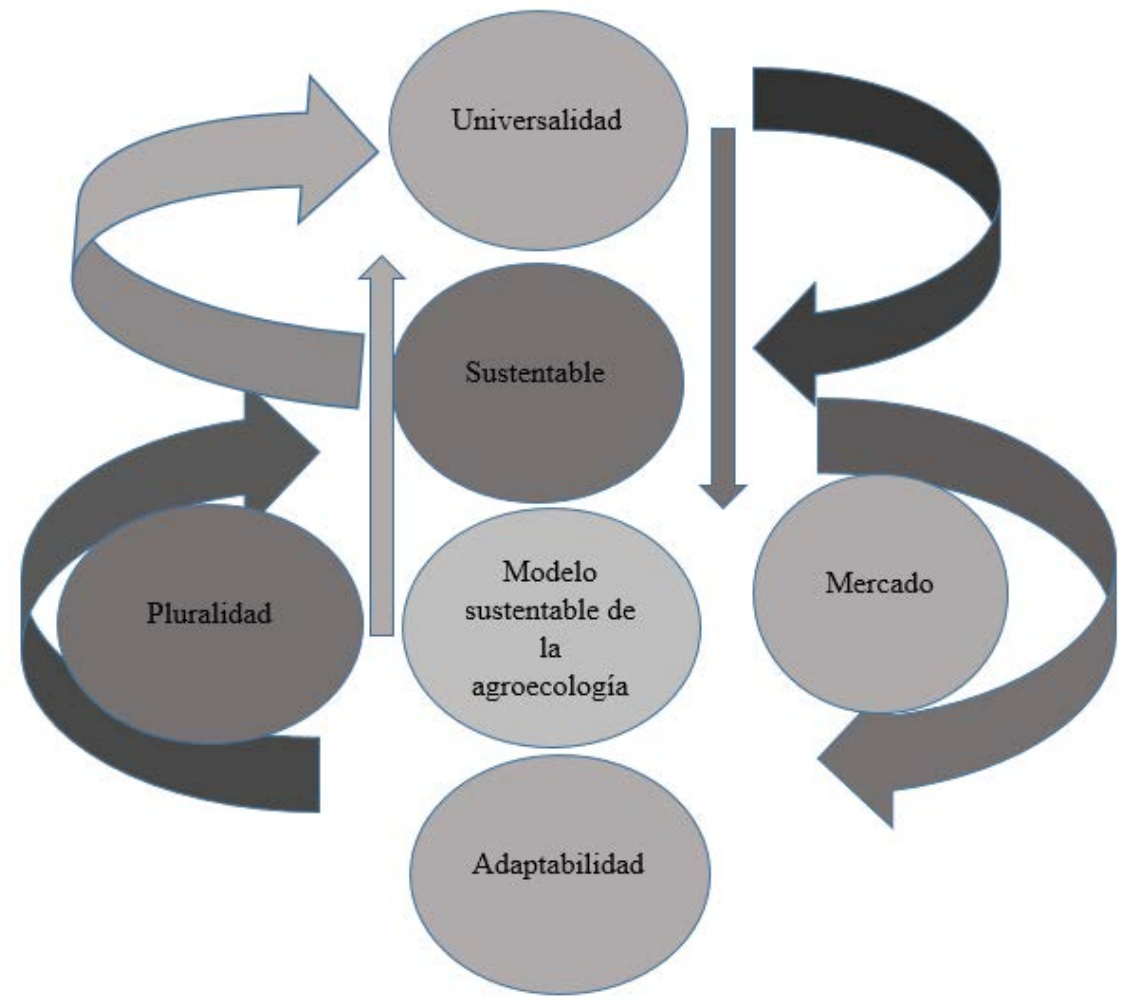


La combinación de las once propuestas, conjuga nuestro planteamiento de las mediciones excluidas en esta ciencia, para la viabilidad del sector agropecuario, así mismo unifica términos y criterios de la ciencia agroecológica como una ciencia amplia y verdaderamente integradora de los procesos productivos modernos.

Los seres humanos hemos comenzado a buscar formas de practicar la agricultura moderna mientras reducen los impactos negativos en el medio ambiente. A medida que avanzamos hacia el futuro con la demanda de alimentos, la agricultura debe satisfacer esas demandas y al mismo tiempo, reducir sus impactos externos en el medio ambiente.

Esta nueva ciencia, podría mejorar la viabilidad económica de las operaciones agrícolas a través de precios superiores para los productos agroecológicos, - podría reducir la viabilidad económica de las operaciones agrícolas atrapando a la mayoría de los agricultores de pequeña escala y de escasos recursos en la pobreza.

La agroecología podría mejorar la calidad de vida de los agricultores que se identifican ideológicamente con ella, o podría empeorar su calidad de vida al no brindarles una salida a la pobreza. Sin embargo, si logramos unificar y practicar estas once longitudes, estaremos avanzando aún más en hacer de las ciencias agroecológicas un camino de solución permanente a la crisis que atraviesa la agricultura.

Las tecnologías agroecológicas no enfatizan el aumento de los rendimientos en condiciones óptimas como lo hacen las tecnologías de la "Revolución Verde", sino que garantizan la constancia de la producción en una amplia gama de condiciones climáticas y de suelo, y muy especialmente en condiciones marginales, que generalmente prevalecen en la pequeña agricultura.

\section{CONCLUSIONES}

En este documento se soporta desde la perspectiva analítica, práctica y conceptual, la oportunidad de ampliar las medidas, que a nuestro criterio son excluidas de la agroecología como alternativa para la sostenibilidad de los sistemas de producción, dentro de estas dimensiones resaltamos la universalidad que debe de poseer esta disciplina como ciencia y su aplicación práctica para los modelos de producción modernos.

Entre las magnitudes de mayor relevancia y que en su conjunto componen y hacen de esta disciplina una ciencia práctica, es la adaptabilidad y la 
sustentabilidad, estas dos de las cinco propuestas son necesarias para el desarrollo de cualquier economía de un país.

Consecuentemente cualquier planteamiento aplicado que sustente a esta iniciativa como la salida a los problemas de la agricultura debe de estar rigurosamente medido para afianzar la economía, para sustentar esos principios ecológicos.

Por eso y bajo esta perspectiva, se sugiere que esta ciencia no debería ser sujeta de exclusiones de ninguna índole, y dado que es una iniciativa multidisciplinaria está en la obligación de integrar las ciencias y tecnologías modernas para el desarrollo de la agricultura actual.

En materia propositiva e integral aportar ideas y planteamientos para conjugar y aplicar más las medidas que esta materia excluye, pues es de alta importancia y relevancia, debido a que metodológicamente si se pueden integrar más alcances y quizás más profundas al sistema agroecológico.

A medida que esta ciencia haciendo más práctica y menos teórica, aunque en este sentido muchos agroecológos de la actualidad y críticos de dicha ciencia, consideran que la materia aun no goza de la legitimidad para luchar contra un sistema alimentario industrial que si la tiene.

Los aspectos propuestos relativamente conjugan el proceso práctico de cualquier ciencia y sobre todo la que está siendo sujeta de revisión, que quiero mencionar con esto, que para que la disciplina sea más convincente debe de graduarse bajo los principios de la agricultura sustentable.

Las magnitudes como la conexión con el mercado, la no reducción de áreas de producción, son elementos importantes que no demos de obviar. Porque de nada serviría reducir las áreas de producción para aplicar agroecología ante el inminente crecimiento poblacional.

Acoger y aplicar los principios agroecológicos con las mejoras de las dimensiones planteadas en este artículo, requiere de grandes retos y de una buena conciencia, que involucre desde los gobiernos centrales, municipales y locales.

A si mismo debe de integrar instituciones del estado, que trabajen directamente en el sector agropecuario, no podemos olvidar los organismos no gubernamentales que trabajan con productores, las universidades que imparten ciencias aplicadas y modernas también son fundamentalmente esenciales en la aplicación de las dimensiones propuestas.

La viabilidad de las aplicaciones prácticas de las ampliaciones planteadas en este ensayo para una agroecología incluyente a corto plazo, simboliza una gran oportunidad, no solo para mejorar los procesos productivos sustentables, 
si no para garantizar la seguridad alimentaria de una población creciente y demandante de alimentos sanos.

\section{REFERENCIAS BIBLIOGRÁFICAS}

Altieri, M.A. (1987). Agroecología: La base científica de la agricultura alternativa. Westview Press, Boulder, Colorado: 227 págs.

Altieri, Miguel A., \& Peter Rosset. (1995). La agroecología y la conversión de los métodos convencionales a gran escala sistemas de gestión sostenible. Revista Internacional de Estudios Ambientales 50: 165-185.

Altieri, M., \& P. Koohafkan (2008). Granjas duraderas: cambio climático, pequeños agricultores y comunidades agrícolas tradicionales. Red del Tercer Mundo, Malasia: p. 72.

Altieri, M. Á., \& Nicholls, C. I. (2012). Agroecología: única esperanza para la soberanía alimentaria y la resiliencia socioecológica. Agroecología, 7(2), 65-83

Caporal, F. R., \& Costabeber, J. A. (2004). Agroecología y extensión rural: contribuciones a la promoción del desarrollo rural sostenible. Brasilia: MDA / SAF / DATER-IICA.

CMMAD (Comisión Mundial del Medio Ambiente y del Desarrollo), (1988). Nuestro futuro común. Madrid: Alianza Editorial.

Dalgaard, T. Hutchings, N. J., \& Porter, J. R. (2003). Agroecología: escalamiento e interdisciplinariedad. Agricultura, ecosistemas y medio ambiente. 100 (1), 39-51.

De Schutter, O. (2010). Consejo de Derechos Humanos, Informe Especial sobre el derecho a la alimentación, U.N. Doc. A / HRC / 16/49, recuperado de: http://www2.ohchr.org/english/issues/food/docs/A-HRC-16-49.pdf

De Schutter., \& Ga“eten Vanloqueren (2011). La nueva revolución verde: cómo la ciencia del siglo XXI puede alimentar al mundo, Soluciones. págs. 33-44.

FAO (Organización de las naciones unidas para la alimentación y la agricultura), La FAO pide un "cambio de paradigma" hacia la agricultura sostenible y la agricultura familiar. 201. Autor. Recuperado de: http:// www.fao.org/news/story/en/item/250148/icode/ (consultado el 13 de octubre de 2020).

Francis, C. Lieblein, G., Gliessman, S., Breland, T. A., Creamer, N., Harwood, R., \& Wiedenhoeft, M. (2003). Agroecología: la ecología de los sistemas alimentarios. Revista de agricultura sostenible, 22 (3), 99-118.

Garnett, T., \& Godfray, C. (2012). Intensificación sostenible de la agricultura: Navegar por un curso a través de las prioridades del sistema alimentario en competencia. Red de investigación sobre el clima de los alimentos y el programa Oxford Martin sobre el futuro de los alimentos, Universidad de Oxford, Reino Unido: 51. 
Guzmán Casado, G. I., González de Molina Navarro, M., \& Sevilla Guzmán, E. (2000). Introducción a la agroecología como desarrollo rural sostenible (No. Sirsi) i9788471148704).

Guzmán, E., \& Woodgate, G. (2013). Agroecología: Fundamentos del pensamiento social agrario y teoría sociológica. Agroecol. Sostener. Food Syst. 37, 32-44.

Gliessman, S (2013). Agroecología: Cultivando las raíces de la resistencia. Agroecol. Sostener. Food Syst., 37, 19-31. 8. Altieri, M.A. Nicholls, C.I. Ampliación de la agroecología para la soberanía alimentaria y la resiliencia. Sostener. Agric. Rev. 2012, 1-29. [CrossRef]

Hewitt, T. I., \& Smith, K. R. (1995). Agricultura intensiva y calidad ambiental: examinando el mito agrícola más reciente. Instituto Henry A. Wallace de Agricultura Alternativa:

IICA (Instituto interamericano de cooperación para la agricultura), (1999). Discurso de Severino De Melo Araujo, Subdirector General de FAO para América Latina y el Caribe: XI Conferencia Latinoamericana de ALEAS. Abril 1997. Santiago, Chile: En: Educación Agrícola Superior, Desarrollo Sostenible, Integración Regional y Globalización, R Chateneuf, A Violic \& E Paillacar (Eds): 9-13. Autor.

IICA (Instituto interamericano de cooperación para la agricultura), (2012). Situación y desempeño de la agricultura en América Latina, desde la perspectiva tecnológica. San José, C.R: IICA. 92 pp Autor.

Keller, D. (2013). Muestra de conservación y Mayordomía: Agricultura Progresiva.pág. 42.

Kershen, D. L. (2006). Agricultura intensiva sostenible: alta tecnología y beneficios ambientales. Kan. JL y Pub. Pol'y, 16, 424.

López-García, D., Calvet-Mir, L., Espluga, J., Di Masso, M., Tendero-Acin, G., \& Pomar-León, A. (2015). La dinamización local agroecológica como estrategia para la construcción de soberanías locales: Ecología Política. (49), 28-34

Mc Ginn, AP. (2000a). ¿̇Por qué envenenarnos a nosotros mismos?: Un enfoque de precaución para los productos químicos sintéticos. Chris Bright, editor, Worldwatch Paper 153: 92 págs.

Mc Ginn, AP. (2000b). Eliminación de contaminantes orgánicos persistentes: en estado del mundo 2000, Informe del Instituto Wordlwatch sobre el progreso hacia una sociedad sostenible. WW Norton \& Company, Nueva York-Londres: 80-100.

Mockshell, J., \& Kamanda, J. (2018). Más allá del debate sobre la intensificación agrícola agroecológica y sostenible: ¿̇es la sostenibilidad combinada el camino a seguir ? Revista Internacional de Sostenibilidad Agrícola, 16 (2), 127-149.

Nosty, B. D. (2009). Cambio climático: consenso científico y construcción mediática. Los paradigmas de la comunicación para la sostenibilidad. Revista Latina de comunicación social, 12(64), 99-119.

Pérez-Vázquez, A., \& Ruiz-Rosado, O. (2005). Investigación interdisciplinaria: un análisis DAFO y su papel en la investigación agrícola en México: Agroecosistemas tropicales y subtropicales, 5 (3), 91-99. 
PNUMA (Programa de las Naciones Unidas Para el Medio Ambiente), (1990). Reseña del PNUMA.Nairobi, Kenia: 48 pp. Autor.

Quist, et al. (2013). Hambriento de innovación: Vías de cultivos transgénicos Agroecología, 2 Lecciones Tardias de las advertencias tempranas: $\mathrm{SCl}$., Precaución, Innovación 490, 508.

Rosset, P. M., \& Altieri, M. A. (1997). Agroecología versus sustitución de insumos: una contradicción fundamental de la agricultura sostenible. Sociedad y recursos naturales, 10 (3), 283-295.

Sarandón SJ, MS Zuluaga, R Cieza, C Gómez, L Janjetic., \& E Negrete (2006). Evaluación de la sustentabilidad de sistemas agrícolas de fincas en Misiones, Argentina: mediante el uso de indicadores. Revista Agroecología, 1: 19-28.

Sarandón, S.J., \& C.C. Flores. (2014). Análisis y evaluación de agroecosistemas: Construcción y aplicación de indicadores. pp. 375-410. En: Sarandón, S.J. y C.C. Flores (eds.). Bases teóricas para el diseño y manejo de agroecosistemas sustentables. Universidad Nacional de La Plata, La Plata: Argentina.

Sarandón, S. J., \& Flores, C. C. (2014b). Agroecología. Editorial de la Universidad Nacional de La Plata: (EDULP).

Tranquilli, C. A. (2015). Necesidad y costes de una política que transforme la agricultura convencional en orgánica. (Doctoral disertación, Universidad de Guayaquil: Facultad de Ciencias Económicas)

Tribi, Derek. (1994). Alimentar y ecologizar el mundo: el papel de la investigación agrícola internacional. Wallingford, Inglaterra: $C A B$ International.

Wezel, A., \& Soldat, V. (2009). Un análisis histórico cuantitativo y cualitativo de la disciplina científica de la agroecología. Revista internacional de sostenibilidad agrícola, 7 (1), 3-18.

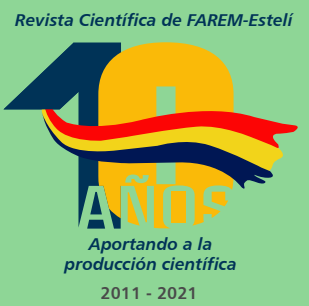

